\title{
Globalisation of clinical trials and ethics of benefit sharing
}

The Declaration of Helsinki ${ }^{1}$ holds that if medical research is done within a vulnerable group, this group should stand to benefit from the knowledge, practices, or interventions resulting from the research. This principle, reaffirmed by all versions of the Declaration released in this millennium, represents the need for a direct link between obtaining scientific results and achieving health benefits in vulnerable groups. ${ }^{1}$

Vulnerability is generally defined as having diminished autonomy and increased likelihood of incurring harm or exploitation. On the basis of the rights to integrity and autonomy, vulnerable people should receive assistance to enable them to realise their potential. In medical research, vulnerability is often perceived in relation to individuals who are minor of age, institutionalised, or incapacitated on medical grounds. However, vulnerability goes beyond juridical, cognitive, and medical boundaries. For instance, the Belmont Report includes minorities and the economically disadvantaged in their classification of vulnerable groups. In poor countries, vulnerability is often linked to poverty, social exclusion, and poor access to health care. This relation might drive populations towards participation in clinical trials as a way to secure access to free health care, which would be otherwise unavailable.

The ethical guidelines for medical research by the Council for International Organizations of Medical Sciences tackle the issue of research in socioeconomically vulnerable communities. ${ }^{2}$ In line with the Helsinki Declaration and the principles of solidarity and non-discrimination, the guidelines unequivocally state that any product developed in a research programme should be made available to the population involved in that research. If there are reasons to believe that these conditions will not take place, the guidelines deem it unethical and exploitative to do the research in that country. To share the burdens and benefits of research fairly, innovative drugs showing superiority over the standard of care should become accessible and affordable to prospective patients residing in the study countries within a reasonable time frame.

The development of costly life-saving drugs against non-communicable diseases through trials done in middle-income countries (MICs) poses ethical challenges that, to our knowledge, have not been investigated in depth. MICs are defined by the World Bank as having a yearly gross national income (GNI) per head greater than US\$1045 and less than $\$ 12746$. $^{3}$ Haematological malignancies might be a useful setting in which to monitor the benefits and ethical implications of clinical trials in MICs, since they are ubiquitous and drugs exist that either cure or permit long-term control in a substantial number of patients. The downside of these drugs, whether they are recombinant monoclonal antibodies or small molecules that target specific molecular pathways, is their high cost, which might be unsustainable in MICs.

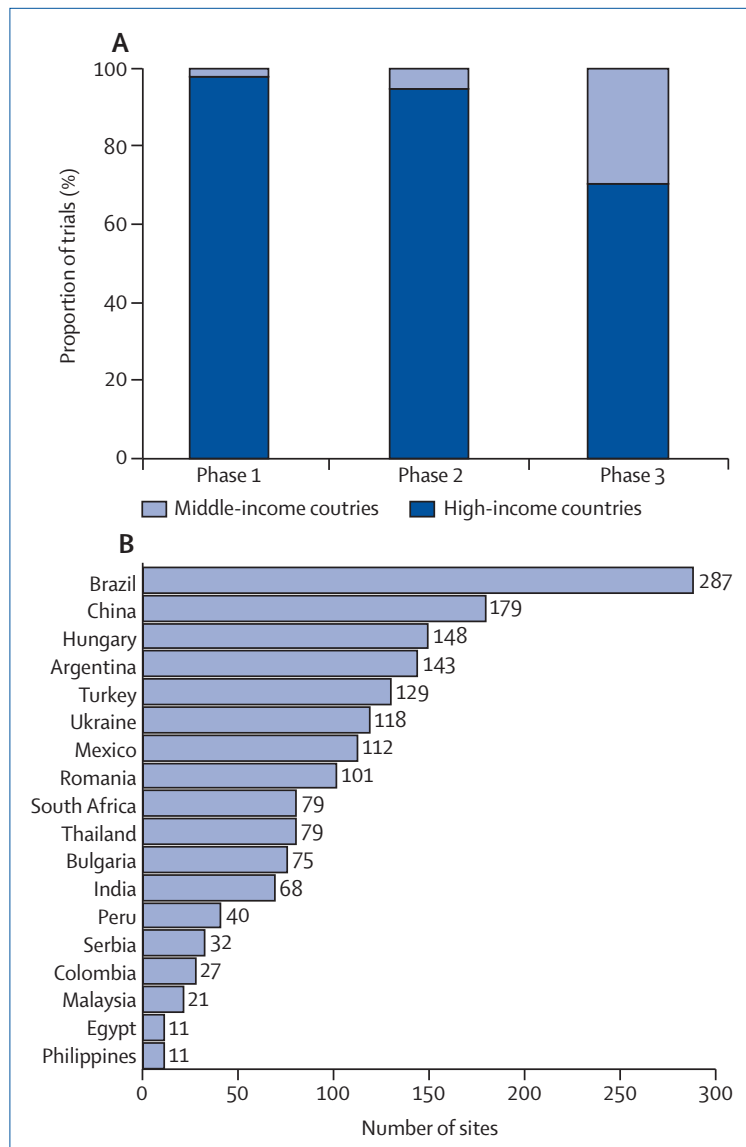

Figure: Involvement of middle-income countries in clinical trials for haematologic malignancies

(A) Proportion of interventional, industry-sponsored clinical trials run in MICs for leukaemia, lymphoma, and multiple myeloma. (B) Clinical trial sites in MICs included in studies testing 12 new drugs against leukaemia, lymphoma, or multiple myeloma $(n=73)$. Only MICs with more than ten study sites are shown. MICs with less than ten study sites include Albania, Algeria, Belarus, Bosnia Herzegovina, Dominican Republic, Ecuador, El Salvador, Georgia, Guatemala, Indonesia, Macedonia, Montenegro, Morocco, Panama, Tunisia, Venezuela, and Vietnam. MIC=middle-income country. $\mathrm{HIC}=$ high-income country.
For Belmont Report see hhs. gov/ohrp/humansubjects/ guidance/belmont.html 
As an example, the cost of treating chronic myeloid leukemia with a first generation small molecule drug exceeds $\$ 40000$ per patient per year in South Africa. ${ }^{4}$ Such an expense is more than 130 times the yearly per head government expenditure rate on health care (\$309 in 2012) and about six times the GNI per head (\$7190 in 2013). ${ }^{3.5}$ In a high-income country like Italy, the yearly cost for the same condition would be 13 times the yearly per head government expenditure rate on health care, and 1.1 times the GNI per head. ${ }^{3-5}$ In MICs, accessibility to health care is further reduced because of high poverty, high out-of-pocket payments, and low health protection coverage. According to the International Labour Organization, of $58 \mathrm{MICs}$ for which data are available, 39 have a medium-to-very-high level of vulnerability, with proportions of the population not covered by any legal health protection ranging from 30.8-88.4\%. ${ }^{6}$ For most people in these countries, treating haematological malignancies with costly drugs might represent a catastrophic expense, pushing patients and families into extreme poverty.

Trials for haematological malignancies have followed a trend for globalisation that is characteristic of clinical research in the past years. ${ }^{7,8}$ Of all phase 3 , interventional, industry-sponsored clinical trials with innovative drugs against leukaemia (1228 trials), lymphoma (1344 trials), or multiple myeloma (610 trials) that have been submitted to ClinicalTrials.gov (as of July 14, 2014), about $30 \%$ involved sites in MICs, including both lowmiddle-income and upper-middle-income countries (figure). We defined MICs according to the World Bank classification (2015 fiscal year), which might present minor differences to the classification at the time the various studies were started.

We analysed in greater detail the studies of 12 drugs recommended by international guidelines or which are under development (eight small molecules: bortezomib, carfilzomib, dasatinib, ibrutinib, idelasilib, imatinib, lenalidomide, and nilotinib; and four monoclonal antibodies: brentuximab, obinutuzumab, ofatumumab, and rituximab; figure). We identified 73 international, industry-sponsored studies (four phase 1; 19 phase 2; 50 phase 3) involving MICs in ClinicalTrials.gov. When considering all 9559 study sites, the proportion of sites in MICs was $18.0 \%$ (range 1.6-79.0). When restricting the analysis to sites located outside USA ( $n=8142)$, $21 \cdot 1 \%(2 \cdot 1-79 \cdot 0)$ were in MICs. The highest proportion of MIC sites in any trial (79.01\%) was in a phase 3 trial, whereas the proportion of MIC sites in phase 1 and 2 trials did not exceed $25.8 \%$ and $33.7 \%$, respectively. Trials run in MICs targeted all age groups, including children, adolescents, and elderly people.

A peculiar issue occurs in phase 3 trials that compare costly, life-saving drugs close to patent expiration with a second-generation compound developed by the same patent holder. Such trials have been done in MICs even though the control drug had never been widely accessible, despite its common use for more than a decade in affluent countries. These trials might raise a substantial ethical issue, because neither the comparator nor the investigational compound have had or will have a reasonable chance of becoming accessible to all those in need in the study country.

Overall, the drive for globalisation of clinical trials of costly drugs adds a further degree of complexity to the ethical concerns surrounding trials that might be delocalised for administrative convenience, as highlighted in the Belmont Report. Moving them, at least partly, to MICs could shorten the timelines of clinical development, thanks to a combination of lower research costs, availability of larger pools of potential participants, and less stringent oversight. ${ }^{9}$

The shift in geographical boundaries of trials with costly drugs should prompt a quest for ethically acceptable solutions, rather than being perceived as an obstacle toward MIC involvement. An active role of MICs in research is beneficial if a reasonable likelihood that their populations will benefit from the research exists. Several strategies might be explored. First, scientific journals should publish a complete list of the trial sites and the number of cases per site, in line with the CONSORT guidelines that require information on settings and locations where the data were collected. Second, when discussing the clinical development plan of a potentially life-saving drug, regulatory agencies should require an ethical clause that binds the marketing authorisation holders to register it in all MICs involved in the trials, to make it available at tiered prices based on the GNI of MICs, and to make such information publicly available. This will require the proactive involvement of local regulatory authorities. Third, physicians and patients from MICs, hopefully joined by their peers in affluent countries, should lobby for a fair price for the drugs tested in their countries. ${ }^{4}$ The implementation 
of these and other measures will only succeed if grounded in a global collaborative effort and will foster the equitable distribution of burdens and benefits of participation in research. Such effort would contribute to filling the persisting gaps in access to life-saving therapies for non-communicable diseases. ${ }^{10}$

\section{Raffaella Ravinetto, Pier Davide Guenzi, Pierre Massat, ${ }^{*}$ Gianluca Gaidano}

Clinical Sciences Department (RR), Public Health Department (PM), Institute Tropical Medicine Antwerp, Antwerp, Belgium; Clinical Pharmacology and Pharmacotherapy Department, KU Leuven, Leuven, Belgium (RR); Catholic University of the Sacred Heart, Milan, Italy (PDG); and Division of Haematology, Department of Translational Medicine, Amedeo Avogadro University of Eastern Piedmont, 28100 Novara, Italy (GG) gaidano@med.unipmn.it

RR, PDG, and PM have nothing to disclose. GG reports personal fees outside the submitted work from Roche, Janssen, Amgen, Novartis, Bristol-Myers Squibb, and grants from Celgene.

1 World Medical Association. Declaration of Helsinki: ethical principles for medical research involving human subjects. JAMA 2013; 310: 2191-94

2 International Ethical Guidelines for Biomedical Research Involving Human Subjects, Council for International Organizations of Medical Sciences (CIOMS) in collaboration with the WHO. Geneva: CIOMS 2002. http://www. cioms.ch/publications/guidelines/guidelines_nov_2002_blurb.htm (accessed July 22, 2014)
3 The World Bank. Country and Lending Groups, data for fiscal year 2015 http://data.worldbank.org/about/country-and-lending-groups (accessed July 22, 2014)

4 Experts in Chronic Myeloid Leukemia. The price of drugs for chronic myeloid leukemia (CML) is a reflection of the unsustainable prices of cance drugs: from the perspective of a large group of CML experts. Blood 2013; 121: 4439-42.

5 World Health Organization. http://apps.who.int/gho/data/node. main.78?lang=en (accessed 22 July, 2014)

6 International Labour Organization. World Social Security Report 2010/2011: Providing coverage in terms of crisis and beyond. International Labour Office, Geneva. http://www.ilo.org/wcmsp5/groups/public/-dgreports/---dcomm/---publ/documents/publication/wcms_146566.pdf (accessed July 22, 2014)

7 Levinson DR. US Department of Health and Human Services, Office of Inspector General. Challenges to FDA's ability to monitor and inspect foreign clinical trials. 2010, OEl-01-08-00510. http://oig.hhs.gov/oei/ reports/oei-01-08-00510.pdf (accessed July 22, 2014)

8 European Medicines Agency. Reflection paper on ethical and GCP aspects of clinical trials of medicinal products for human use conducted outside of the EU/EEA and submitted in marketing authorisation applications to the EU Regulatory Authorities. 2012, EMA/121340/2011. http://www.ema. europa.eu/docs/en_GB/document_library/Regulatory_and_procedural guideline/2012/04/WC500125437.pdf (accessed July 30, 2014)

9 Glickman SV, McHutchison JG, Peterson ED, et al. Ethical and scientific implications of the globalization of clinical research. N EngJ Med 2009; 360: 816-23.

10 Bollyky TJ. Access to drugs for treatment of non-communicable diseases PloS Med 10: e1001485. 